\title{
Spontaneous versus Reinforced Olfactory Discriminations
}

\author{
Christiane Linster, ${ }^{1}$ Brett A. Johnson, ${ }^{2}$ Alix Morse, ${ }^{1}$ Esther Yue, ${ }^{1}$ and Michael Leon ${ }^{2}$ \\ ${ }^{1}$ Department of Neurobiology and Behavior, Cornell University, Ithaca, New York 14853, and ${ }^{2}$ Department of \\ Neurobiology and Behavior, University of California, Irvine, California 92697-4550
}

\begin{abstract}
When the major response domains in the rat olfactory bulb that are evoked by odorant enantiomers are compared, some of these odorant pairs do not show significantly different activity patterns. Such pairs are not spontaneously discriminated in a behavioral test. We show here that even these similar odorants appear to evoke different activity patterns when every data point in a glomerular activity array is compared. These odorants also can be discriminated if they are subjected to differential reinforcement. These data suggest that the method chosen to assess olfactory discrimination will reveal different olfactory
\end{abstract}

capabilities of rats. The small differences in glomerular activity that probably exist between any pair of odorants may serve as a basis for odor discrimination when rats are differentially reinforced, thereby establishing the remarkable limits of rat olfactory perception. At the same time, the major differences in glomerular responses appear to serve as the normal basis for spontaneous odor discrimination.

Key words: olfactory coding; enantiomers; optical isomers; odor perception; neural representations; habituation; reinforcement learning; olfactory bulb; glomeruli
Part of understanding the olfactory code requires one to be able to predict olfactory perceptions based on neural representations. To test such predictions, it would be ideal to use pairs of odorants that have a single difference in molecular structure that would evoke a small difference in neural activity. One could then determine if there was a perceptual difference between the two odorants. A good candidate for such molecules are enantiomers, which are pairs of odorants that differ only in their stereoconfiguration. Some, but not all enantiomer pairs evoke different olfactory perceptions (Friedman and Miller, 1971; Leitereg et al., 1971; Laska and Teubner, 1999; Laska et al., 1999a,b; Laska and Galizia, 2001; Rubin and Katz, 2001).

We previously reported that the rat olfactory glomerular responses evoked by the enantiomers of carvone differed significantly, whereas those evoked by enantiomers of limonene and terpinen-4-ol did not (Linster et al., 2001). These data predicted that rats would discriminate between the enantiomers of carvone but not between the enantiomers of either limonene or terpinen4-ol, and we observed exactly that behavior pattern (Linster et al., 2001). Such data are of interest because they show that neural responses can be used to predict the perceptual qualities of odorants. These data also are of interest because they are the first to indicate that rats fail to discriminate between any two odorants and that the olfactory bulb response does not differ between any two odorants.

However, we used a novel means to record the behavior of rats to access their perceptions. Specifically, we habituated rats to the $(-)$-enantiomer and then determined whether the rats dishabituated to the $(+)$-enantiomer or to other odorants (Linster et al., 2001). Dishabituation indicated to us that the rats regarded the

\footnotetext{
Received March 1, 2002; revised May 31, 2002; accepted June 6, 2002.

This work was funded by National Institute on Deafness and Other Communication Disorders Grant DC03545 (M.L.).

Correspondence should be addressed to Christiane Linster, Department of Neurobiology and Behavior, W249 Mudd Hall, Cornell University, Ithaca, NY 14853. E-mail: CL243@cornell.edu.

Copyright (ㄷ) 2002 Society for Neuroscience $0270-6474 / 02 / 226842-04 \$ 15.00 / 0$
}

test odor as different from the original odor. This method elicits a spontaneous response based on initial olfactory perception and thereby differs from the more commonly used differential reinforcement of one odorant over another to determine whether the odors can be discriminated after training (Rubin and Katz, 2001; Slotnick, 2001).

We also used a novel means of comparing the olfactory bulb responses to the odorants (Linster et al., 2001). Specifically, we compared the maximal 2-DG uptake within previously identified glomerular response domains, or modules, that have shared responses to odorants (Johnson and Leon, 2000). This method differs from the more common qualitative reports noting visible differences in glomerular responses to odorant enantiomers in individual animals (Ma and Shepherd, 2000; Rubin and Katz, 2001). One disadvantage of our previous analysis was that it combined many individual measurements into a few response modules for a simplified statistical comparison. Thus, the analysis may have overlooked reliable differences involving areas considerably smaller than the previously defined glomerular modules. We here show that if we compare every data point in a glomerular activity array, rather than focusing on modular analysis, we can measure differences in the glomerular activity maps evoked by the enantiomers of limonene and terpinen-4-ol. Given such potential differences, we predicted that rats would learn to discriminate these odorants in a behavioral paradigm using differential reinforcement.

\section{MATERIALS AND METHODS}

Odorants. Enantiomers of limonene, along with propanal and ethyl isovalerate, were purchased from Aldrich Flavors and Fragrances (Milwaukee, WI). Enantiomers of carvone and terpinen-4-ol were purchased from Fisher Scientific/Acros Organics (Pittsburgh, PA). Purities reported by the manufacturers were $98 \%$ for $(R)-(-)$-carvone, $98 \%$ for $(S)-(+)$ carvone, $95+\%$ for $(S)-(-)$-limonene, $97+\%$ for $(R)-(+)$-limonene, $97 \%$ for $(R)-(-)$-terpinen-4-ol, $95 \%$ for $(S)-(+)$-terpinen-4-ol, $98 \%$ for ethyl isovalerate, and $97 \%$ for propanal.

Odorant exposures. The University of California, Irvine, and Cornell University Animal Care and Use Committees approved all procedures 
involving animals. Groups of six male Wistar rat pups (postnatal days 19-22) received a subcutaneous injection of 2-DG (0.16 mCi $/ \mathrm{kg}$; Sigma, St. Louis, MO) immediately before a 45 min odorant exposure. From each litter, two rats were used for a given enantiomer pair. Limonene and terpinen-4-ol enantiomer exposures involved the same litters, and a single additional rat from each of these litters was exposed to the odorant vehicle as a control.

Odorant exposures for 2-DG uptake were conducted as reported previously (Johnson et al., 1999). Odorants were volatilized by using high-purity nitrogen gas bubbled through a $100 \mathrm{ml}$ column of pure liquid in a gas washing bottle at a flow rate of $250 \mathrm{ml} / \mathrm{min}$. The nitrogen stream then was mixed with ultra zero-grade air for a final flow rate of $21 / \mathrm{min}$ (1:8 dilution of odorant vapor). After odorant exposure, rats immediately were decapitated. Brains were frozen rapidly in 2-methylbutane at about $-45^{\circ} \mathrm{C}$ and then stored at $-70^{\circ} \mathrm{C}$ until sectioning.

Mapping of 2-DG uptake. Coronal sections (20- $\mu \mathrm{m}$-thick) were prepared with a cryostat. Every sixth section was taken for autoradiography, and adjacent sections were stained with cresyl violet as described previously (Johnson et al., 1998, 1999). The stained sections were used both to direct sampling within the glomerular layer of the autoradiography section and to standardize the rostral-caudal position of sections in reference to anatomical landmarks (Johnson et al., 1999). Discrete measurements of 2-DG uptake were directed by a set of radial grids to give samples at $\sim 120 \mu \mathrm{m}$ intervals around each section. Measurements were merged into standardized data arrays covering the entire glomerular layer (Johnson et al., 1999). Arrays from the two bulbs of each animal were averaged and then converted to nanocuries per gram of 2-DG by using standards exposed to the autoradiography films. Values in these arrays then were transformed into $z$ scores before statistical analyses (Johnson et al., 1999).

Statistical analyses. Comparisons of patterns of 2-DG uptake were made to examine small differences in 2-DG uptake pattern by performing $t$ tests at each of the 2500 locations of $z$ score-standardized arrays (Johnson et al., 1999). This procedure is similar to standard analyses used in functional brain mapping (Hess et al., 2000; Crespo-Facorro et al., 2001; Rubin and Katz, 2001). We then constructed a rolled-out map of $p$ values to describe the most reliable differences in 2-DG uptake across the glomerular layer. Any point within this data array is approximately aligned with respect to its position within the bulb, but the absolute size or position of any individual response within the glomerular layer is not revealed by this array.

Olfactory discrimination. All behavioral training occurred in a transparent Plexiglas chamber $(51 \times 38 \times 25 \mathrm{~cm})$ divided with a sliding, opaque Plexiglas panel into a start area and a test area (Linster and Hasselmo, 1999; Linster and Smith, 1999; Cleland et al., 2002). Ceramic bowls ( $9 \mathrm{~cm}$ diameter, $4.5 \mathrm{~cm}$ height) were used to place the odorants and the reward together. At the beginning of each daily training set, a cotton swab was saturated with a $0.1 \mathrm{ml}$ drop of diluted odorant. The cotton tip was covered with fine plastic mesh and was then taped to the bottom of the bowl. The bowl then was filled with bedding (Bed-O-Cobs 0.125 inch laboratory bedding). The reward, a bit of sweetened cereal (Froot Loops; Kellogg's, Battle Creek, MI), was buried in the bedding, which was replaced after every trial.

Shaping. Rats were first shaped to retrieve a reward by digging through the bedding. At the beginning of each trial, the rat was placed in the start area. Two bowls were placed in the test area, one containing both the cereal reward and the odor, the other containing no reward and no odor. When the partition was removed, the rat entered the test area and was allowed to dig in the bowls until it retrieved the reward. During the first few trials, the reward was placed on top of the scented bowl, but after several successful retrievals, the reward was buried deeper and deeper into the bedding. When the rat learned to dig to retrieve the reward, the bowls were moved around in the test area to force the rat to use the odor to locate the correct bowl. Shaping was considered to be complete when a rat could successfully retrieve a reward that was deeply buried in the scented bedding and when the rat would dig even in the absence of a reward.

Behavioral testing. We determined whether adult male Sprague Dawley rats could learn to discriminate between pairs of isomers: $(+)-$ and $(-)$-limonene, as well as (+)- and (-)-terpinen-4-ol. For comparison, we tested the ability of these rats to discriminate between two pairs of control odorants, ethyl isovalerate (apple odor) and propanal (unpleasant fruit odor), as well as (+)- and (-)-carvone, which we had shown previously to be easily discriminated by rats in our habituation-dishabituation task. Because each odorant was only used once, the same rats

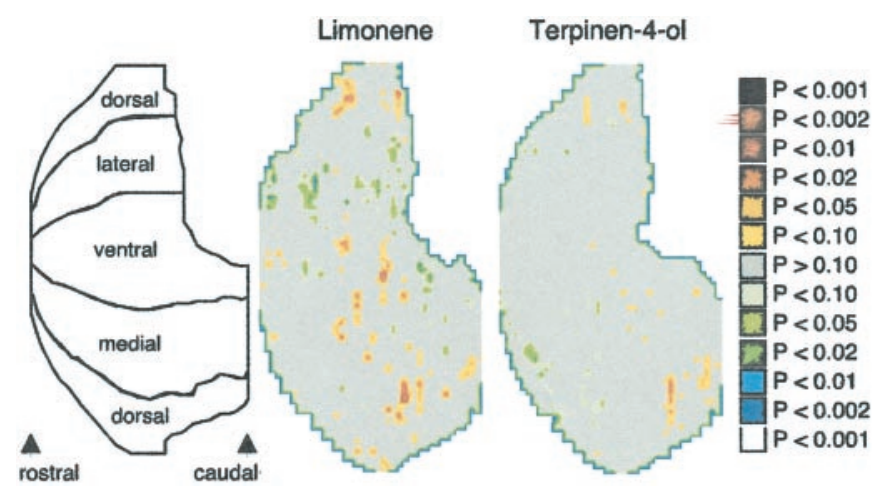

Figure 1. Rolled-out contour charts of the entire glomerular layer indicate the distribution of $p$ values in two-tailed $t$ tests performed between enantiomer responses for both limonene and terpinen-4-ol at each position within the arrays. Warm colors signify locations where the $(-)$-enantiomer evoked potentially greater uptake. Cool colors indicate locations where uptake for the $(+)$-enantiomer potentially exceeded that for the $(-)$-enantiomer.

were used in all experiments. All four discrimination tests were run in parallel, with the order randomized for each rat.

During each trial, rats were presented with two bowls, each containing one of the two enantiomers, but only one of the bowls reliably containing the reward. The presentation of the reward was counterbalanced between odorants. Each training set was comprised of 20 consecutive trials with the same two odorants. During each trial, we recorded the bowl in which digging was first observed, but subjects were left to dig in either bowl until the reward was retrieved. The trial was terminated after $1 \mathrm{~min}$ if the rat did not dig at all. The bedding in the bowls was exchanged after each trial. To ensure that the rats were learning about the test odorants and not identifying the cereal reward by its own smell, we presented the rewarded odorant without the cereal reward on every fifth trial (probe trials). Shortly after the rat registered a preference by digging in the bowl, the reward was dropped onto the scented bedding to maintain the association of the odorant with the reward. Rats that did not dig in either bowl on more than one of these probe trials were excluded from the analysis.

Statistical analysis. Data analyses were performed using SPSS statistical software on the average number of correct responses. During each experiment, some rats had to be excluded because they would not perform the task on that particular day. A total of 11 rats were tested on $(+)$ - and (-)-limonene, 10 rats on $(+)$ and $(-)$-terpinen-4-ol, 13 rats on $(+)$ - and $(-)$-carvone, and seven rats on the control odorant comparison. After two-way ANOVA testing for differences in correct responses to the rewarded odorant, pairwise post hoc tests (Tukey's HSD) were performed to determine which odors induced significant differences in preferences. All tests were two-tailed, and the $\alpha$ level was set at 0.05 .

\section{RESULTS}

When $z$ score-standardized arrays of 2-DG uptake evoked by $(+)$-limonene were compared with those evoked by ( - )-limonene on an individual position-by-position basis, a number of potentially significant $t$ values were obtained (Fig. 1). The low $p$ values often were clustered together, a pattern unlikely to occur on the basis of chance alone. These clusters were found in a scattered distribution across the entire glomerular surface. Clusters of low $p$ values also were obtained in comparisons of patterns evoked by $(+)$-terpinen-4-ol and (-)-terpinen-4-ol. There were locations where (+)-enantiomers evoked higher uptake than (-)enantiomers as well as other areas in which uptake evoked by $(-)$-enantiomers exceeded that evoked by $(+)$-enantiomers. It should be noted that many of these areas of apparently different activity were not obvious by simple inspection of original activity maps, in contrast with our previously reported differences in activity patterns evoked by carvone enantiomers (Linster et al., 2001). 


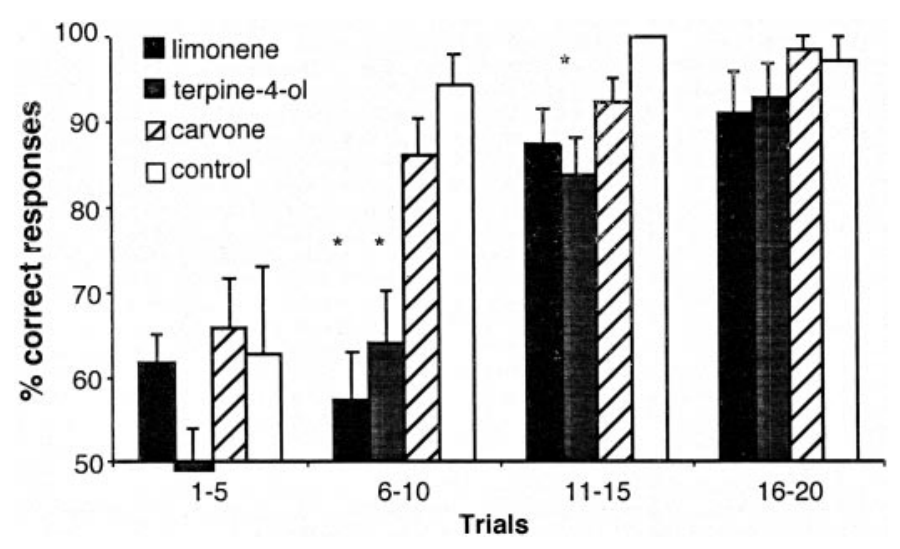

Figure 2. The mean percentage of correct responses to odorants that had previously been rewarded, shown as a function of trial number. Asterisks indicate a response that is significantly different from the response to the control odorants.

Given these small apparent differences in glomerular activity patterns, we considered the possibility that rats might respond differently to these odorants if they were subjected to differential reinforcement. Therefore, we reinforced their selection of one enantiomer over the other with a sweetened food reward (Linster and Hasselmo, 1999). Indeed, rats rapidly reached the learning criterion of $90 \%$ correct responses for discrimination between both limonene enantiomers and terpinen-4-ol enantiomers (Fig. 2). In addition, they learned to discriminate between the enantiomers of carvone and between two unrelated odorants. Although there were no differences among groups in responses during the first five reinforced discriminations, during trials 6-10, significant differences emerged across the four-odorant set $\left(F_{(3,37)}=10.191\right.$; $p<0.001)$. These differences arose between the carvones and limonene $(p<0.001)$, between the carvones and terpinen-4-ol $(p<0.02)$, as well as between the two control odorants and both limonene $(p<0.001)$ and terpinen-4-ol $(p<0.005)$. The number of correct responses to the enantiomers of limonene and terpinen-4-ol were not significantly different from each other, nor were those between the enantiomers of carvone and the two control odorants. During trials 11-15, small differences across odorant sets persisted $\left(F_{(3,37)}=3.665 ; p<0.021\right)$, and this difference arose solely between the enantiomers of terpinen-4-ol and the control odors. There were no significant differences observed during the last five trials in overall responses to the four-odorant set. Thus, although rats eventually learned all three enantiomer discriminations, they were slower to learn to discriminate between the enantiomers of limonene or terpinen-4-ol than between the enantiomers of carvone or between the two unrelated odorants.

\section{DISCUSSION}

We previously showed that rats do not discriminate between $(+)$ and (-)-enantiomers of either limonene or terpinen-4-ol in a spontaneous discrimination task (Linster et al., 2001). Here, we report that rats subjected to differential reinforcement of the enantiomers were able to discriminate the same odorant pairs. In our previous modular analysis of glomerular activity patterns, the enantiomer pairs of limonene and terpinen-4-ol appeared to evoke the same pattern of activity (Linster et al., 2001). In contrast, a point-by-point comparison of activity across the entire glomerular layer revealed numerous, small areas of potential difference in the activity patterns evoked by the same enantiomer pairs. Our data are consistent with the idea that rats normally ignore small differences in glomerular activity and use only the major differences in module responses to make spontaneous perceptual judgments. Whereas small differences in glomerular activity normally may be ignored, such small differences can be used to make discriminations if the rats are subjected to the motivational and experiential consequences of differential reinforcement.

Small differences in glomerular activation patterns could arise from low-affinity responses to one odorant that are not present for the other odorant. Small differences in glomerular activity also could result if two odorants were differentially contaminated with other odorants. At some level of analysis, all odorants are contaminated with other odorants. In most studies on olfaction, where odorant purities range from 95 to $99.9 \%$, odorant impurities sum to equal $0.1-5 \%$ of the total mass of the odorant preparation. Normal rats easily can learn to identify a mixture of $0.01 \%$ cineole and $0.5 \%$ amyl acetate when compared with $0.5 \%$ amyl acetate alone (Lu and Slotnick, 1998). Therefore, it is clear that rats can perceive the levels of contaminants that are found in odorants. One may predict that all odorants (even different preparations of the same odorant) will generate patterns of glomerular activity with at least small differences from all other such patterns. Moreover, rats should be able to use such differences to discriminate between all odorants, given differential reinforcement. Indeed, we can find no report that shows rats failing to discriminate between any two odorants when given differential reinforcement. The major modular responses, however, predict that some odorants will be judged to be similar and others judged to be different when rats are asked to make a spontaneous discrimination. In a world in which virtually all odorants are perceived against a variable background of other odorants that are well within the detection range of rats, categorical distinctions must normally be accomplished by ignoring these contaminants. On the other hand, differential reinforcement may allow rats to use minor glomerular responses, or any other differences they can detect, to accomplish the discrimination.

The broadly distributed pattern of small response differences across much of the olfactory bulb glomerular layer also suggests that it may be futile to lesion major foci in the olfactory bulb and predict that even lesioned rats would be able to discriminate between two odorants if they are reinforced for their choice $(\mathrm{Lu}$ and Slotnick, 1998). It seems quite possible that even if all of the major foci were removed (something that has yet to be accomplished), rats would still be able to use small differences that are likely to persist after bulb damage to support a reinforced discrimination.

In many ways, the use of habituation-dishabituation analyses may be superior to the use of differential reinforcement in experiments attempting to understand the olfactory code. Not only does the procedure predict differences in modular response of odorant enantiomers, it also can show a graded similarity in perception of other closely related odorants, thereby allowing more extensive correlations with neurobiological data. Indeed, correlating the differences in perceptual response, as measured by the spontaneous discrimination test, with the major differences in 2-DG patterns generated by aliphatic acids of different carbon number (Johnson et al., 1999) revealed a correlation coefficient of 0.92 (Cleland et al., 2002). The high correlation of the major glomerular modules with spontaneous discriminations suggests that this approach may be a valuable tool for the understanding of the olfactory code. On the other hand, using differential rein- 
forcement to assess the potential impact of the small differences in glomerular responses may inform us about the extraordinary olfactory abilities of rats.

\section{REFERENCES}

Cleland TA, Morse A, Yue EL, Linster C (2002) Behavioral models for odor similarity. Behav Neurosci 116:222-231.

Crespo-Facorro B, Paradiso S, Andreasen NC, O'Leary DS, Watkins GL, Ponto LL, Hichwa RD (2001) Neural mechanisms of anhedonia in schizophrenia: a PET study of response to unpleasant and pleasant odors. JAMA 286:427-435.

Friedman L, Miller JG (1971) Odor incongruity and chirality. Science 172:1044-1046.

Hess A, Stiller D, Kaulisch T, Heil P, Scheich H (2000) New insights into the hemodynamic blood oxygenation level-dependent response through combination of functional magnetic resonance imaging and optical recording in gerbil barrel cortex. J Neurosci 20:3328-3338.

Johnson BA, Leon M (2000) Modular representations of odorants in the glomerular layer of the rat olfactory bulb and the effects of stimulus concentration. J Comp Neurol 409:495-509.

Johnson BA, Woo CC, Leon M (1998) Spatial coding of odorant features in the glomerular layer of the rat olfactory bulb. J Comp Neurol 393:457-471.

Johnson BA, Woo CC, Hingco EE, Pham KL, Leon M (1999) Multidimensional chemotopic responses to n-aliphatic acid odorants in the rat olfactory bulb. J Comp Neurol 409:529-548.
Laska M, Galizia CG (2001) Enantioselectivity of odor perception in honeybees (Apis mellifera carnica). Behav Neurosci 115:632-639.

Laska M, Teubner P (1999) Olfactory discrimination ability of human subjects for ten pairs of enantiomers. Chem Senses 24:161-170.

Laska M, Galizia CG, Giurfa M, Menzel R (1999a) Olfactory discrimination ability and odor structure-activity relationships in honeybees. Chem Senses 24:429-438.

Laska M, Liesen A, Teubner P (1999b) Enantioselectivity of odor perception in squirrel monkeys and humans. Am J Physiol 277:R1098-R1103.

Leitereg TJ, Guadagni DG, Harris J, Mon TR, Teranishi R (1971) Evidence for the difference between the odours of the optical isomers $(+)$ - and $(-)$-carvone. Nature 230:455-456.

Linster C, Hasselmo ME (1999) Behavioral responses to aliphatic aldehydes can be predicted from known electrophysiological responses of mitral cells in the olfactory bulb. Physiol Behav 66:497-502.

Linster C, Smith BH (1999) Generalization between binary odor mixtures and their components in the rat. Physiol Behav 66:701-707.

Linster C, Johnson BA, Morse A, Yue E, Xu Z, Hingco EE, Choi Y, Choi M, Messiha A, Leon M (2001) Perceptual correlates of neural representations evoked by odorant enantiomers. J Neurosci 21:9837-9843.

Lu XC, Slotnick BM (1998) Olfaction in rats with extensive lesions of the olfactory bulbs: implications for odor coding. Neuroscience 84:849-866.

Ma M, Shepherd GM (2000) Functional mosaic organization of mouse olfactory receptor neurons. Proc Natl Acad Sci USA 97:12869-12874.

Rubin RD, Katz LC (2001) Spatial coding of enantiomers in the rat olfactory bulb. Nat Neurosci 4:355-356.

Slotnick B (2001) Animal cognition and the rat olfactory system. Trends Cogn Sci 5:216-222. 